\title{
Síntese e Caracterização do Copolímero Poli (Ácido Lático-B-Glicol Etilênico)
}

\author{
Walker S. Drumond, Shu Hui Wang \\ DEMM, Escola Politécnica, USP \\ Cheila G. Mothé \\ Departamento de Tecnologia Orgânica, Escola de Química, UFRJ
}

Resumo: Duas séries de copolímeros triblocos, poli (ácido lático-b-etileno glicol) (PLA-PEO-PLA), foram sintetizados a partir do éster cíclico 1,1-lactideo, usando octoato de estanho como iniciador e poli (etileno glicol) $(\mathrm{PEG})(\mathrm{Mn}=4000 \mathrm{ou}$ 600), di-hidróxi terminado, como co-iniciador. A ligação química entre os segmentos PEG e PLA foi confirmada pelas técnicas de espectroscopia no infravermelho (FTIR) e Ressonância Magnética Nuclear de Hidrogênio (RMN- $\left.{ }^{1} \mathrm{H}\right)$ e análise térmica. O grau de polimerização do bloco PLA, DP ${ }_{\mathrm{PLA}}$, no copolímero foi estimado por RMN- ${ }^{1} \mathrm{H}$ e mostrou variação em função da massa molar do PEG e do aumento da concentração relativa de 1,1 lactídeo na mistura reacional. O aspecto dos copolímeros sintetizados variou de acordo com o tamanho dos blocos hidrofílico e hidrofóbico presentes em sua estrutura. Testes de solubilidade mostraram comportamento anfifílico para o produto sintetizado. Os segmentos de PLA formaram uma fase cristalina enquanto a fase cristalina correspondente aos segmentos PEG não foi observada.

Palavras-chave: Polimerização, poli(ácido lático-b-glicol etilênico), lactídeo, PLA-PEG.

\section{Synthesis and characterization of Poly(lactic acid-b-ethylene glycol) copolymer}

Abstract: Two series of tri-block copolymers, poly(lactic acid-b-ethylene glycol) (PLA-PEG-PLA), were synthesized from 1,1-lactide using stannous di-octoate as initiator and di-hydroxy terminated poly(ethylene glycol) $(\mathrm{PEG})(\mathrm{Mn}=600$ or 4000) as co-initiator. The chemical linkage between the PEG segment and the PLA segments was confirmed by Fourier transform infrared spectroscopy (FTIR), by proton magnetic resonance spectroscopy $\left({ }^{1} \mathrm{H}-\mathrm{NMR}\right)$ and thermal analyses. The polymerization degree of PLA segments in copolymers, $\mathrm{DP}_{\mathrm{PLA}}$, was estimated from ${ }^{1} \mathrm{H}-\mathrm{NMR}$ spectra and has shown a dependence on the PEG molar mass and the relative 1,1-lactide feed concentration. The physical aspect of copolymers changed according to the hydrophilic and hydrophobic segments lengths. The solubility tests revealed the amphiphilic nature of copolymers. Longer PLA segments in copolymers still presented crystallinity while the crystallization of the PEG segments was suppressed.

Keywords: Polymerization, poly(lactic acid-b-ethylene glycol), lactide, PLA-PEG.

\section{Introdução}

Poli(ácido lático) é um poliéster relativamente hidrofóbico, instável em condições úmidas e biodegradável a subprodutos atóxicos (ácido lático, $\mathrm{CO}_{2}$ e $\mathrm{H}_{2} \mathrm{O}$ ), presentes no metabolismo de animais e microorganismos. Portanto, apresenta potencial para aplicações nas áreas médicas e farmacêuticas, principalmente como dispositivos temporariamente implantáveis (suturas, grampos, nano-reservatórios para drogas etc) ${ }^{[1-3]}$. Entretanto, devido ao seu caráter hidrofóbico, sua degradação in vivo ocorre predominantemente via processos celulares resultantes de reação inflamatória local ${ }^{[4]}$. Durante sua função temporária, os implantes, preferencialmente, não devem estimular respostas adversas (inflamações, alergias, irritações etc ) e devem ser absorvidos em um tempo apropriado à terapia clínica. Em geral, a maioria dos materiais aplicados como dispositivos ortopé- dicos (grampos de fixação e pinos) são totalmente absorvidos e eliminados em aproximadamente um a um ano e meio, devolvendo, gradualmente, neste período, a função mecânica ao tecido ósseo em cicatrização. No entanto, o poli(ácido lático) pode requerer anos para ser completamente degradado e excretado pelo organismo ${ }^{[5]}$.

Através da modificação química do PLA com poli(glicol etilênico), PEG, um copolímero anfifílico é formado, o qual é mais susceptível a degradar por hidrólise, com menor adsorção de proteínas, células, e/ou tecidos e, portanto, menos susceptível a desencadear reações adversas. Além disso, este copolímero também apresenta propriedade autoassociativa em meio aquoso, ou seja, formação de suspensões coloidais (dispersões de sólidos com tamanho de micro/ nanopartículas em líquidos) termodinamicamente metaestáveis. Nano/micro partículas do PLA-PEG-PLA podem permanecer no sistema circulatório por tempos mais

Autor para correspondência: Wang Shu Hui, Escola Politécnica, USP, Rua Prof. Mello Morais n²463 - Butantã, CEP: 05508-900, São Paulo, SP. E-mail: wangshui@usp.br 
longos que nano/micro partículas do homopolímero PLA, devido a suas propriedades de superfície ${ }^{[6-10]}$.

Neste trabalho, descrevemos a síntese de duas séries de PLA-PEG-PLA, cujos segmentos PEG apresentam $\mathrm{M}_{\mathrm{n}}$ igual a 600 (líquido) ou a 4000 (sólido). Os copolímeros foram caracterizados quanto a sua composição por FTIR e por RMN${ }^{1} \mathrm{H}$ e algumas características térmicas foram reveladas por TG e DSC.

A rota selecionada, copolimerização a partir do sistema 1,1-lactídeo/PEG/octoato de estanho foi previamente investigada por diversos autores e acredita-se que ocorra pelo mecanismo de inserção por coordenação ${ }^{[11-13]}$.

O entendimento detalhado do mecanismo de poliadição por abertura de anel do monômero cíclico, via inserção por coordenação, é essencial para a seleção dos parâmetros mais adequados a síntese de estruturas diversas, apropriadas a diferentes aplicações como biomateriais. Entre estes fatores podemos citar traços de umidade, impurezas e compostos contendo grupos $\mathrm{OH}$, entre outros; e o co-iniciador é um parâmetro reacional fundamental na definição do mecanismo e da estrutura final do produto ${ }^{[14]}$.

Quando o co-iniciador é uma molécula com múltiplos grupos hidroxila (ex. sorbitol, manitol), um polímero de múltiplos braços pode ser obtido, enquanto o uso de um polímero contendo grupo $\mathrm{OH}$ leva à formação de copolímero em bloco ou enxertado. A massa molar é também função da concentração de co-iniciador. Excesso de umidade reduzem drasticamente a massa molar do PLA obtida via abertura do anel lactideo.

De acordo com Kowalski e colaboradores ${ }^{[13]}$ a polimerização de lactídeos envolve um mecanismo de coordenaçãoinserção em duas etapas quando um co-iniciador está presente.

Na primeira etapa $\mathrm{SnOct}_{2}$ reage com o co-iniciador. Duas reações são possíveis nesta primeira etapa:

$$
\begin{aligned}
& \mathrm{Sn}(\mathrm{Oct})_{2}+\mathrm{ROH} \rightarrow \mathrm{OctSn}-\mathrm{OR}+\mathrm{OctH} \\
& \mathrm{Sn}(\mathrm{Oct})_{2}+\mathrm{ROH} \rightarrow \mathrm{Sn}(\mathrm{OR})_{2}+2 \mathrm{OctH}
\end{aligned}
$$

Na primeira etapa da reação $\mathrm{Sn}(\mathrm{Oct})_{2}$ forma fortes complexos com traços de água, álcool, ou impurezas contendo grupos $\mathrm{OH}$, gerando um alcóxido, o verdadeiro iniciador da reação.

Em uma segunda etapa o monômero cíclico se insere entre a ligação -Sn-OR, promovendo o crescimento da cadeia (propagação). O aumento da concentração de co-iniciador resulta em polímeros com massas molares reduzidas. Kowalski, atribui este comportamento ao duplo papel do coiniciador na formação do iniciador verdadeiro e como agente de transferência, esquematizado na reação (3).

$$
\mathrm{OctSn}(\mathrm{LA})_{\mathrm{n}}-\mathrm{OR}+\mathrm{ROH} \rightarrow \mathrm{OctSnOR}+\mathrm{HO}-(\mathrm{LA})_{\mathrm{n}}-\mathrm{OR}
$$

Onde (LA) $)_{\mathrm{n}}$ representa as unidades repetitivas, por exemplo lactato. $\mathrm{Na}$ ausência de reação de transferência o $M_{n}$ do polímero cresce linearmente com a conversão do monômero.

As duas séries de copolímeros sintetizados a partir do
PEG 600 e 4000, permitiram correlacionar o tamanho relativo dos blocos hidrofílico (PEG) e hidrofóbico (PLA) com as características do material (líquido, ceroso, sólido, hidrofílico etc). Esta correlação é fundamental para projetar as estruturas de copolímeros apropriadas às diversas aplicações médicas (ortopédicos, suturas, veículos de drogas, etc).

\section{Materiais e Métodos}

Materiais: 1,1 lactídeo [(3S)-cis-3,6-dimetil-1,4-dioxano2,5-diona] e 2-etil-hexanoato de estanho $\left[\mathrm{CH}_{3}\left(\mathrm{CH}_{2}\right)_{3}\right.$ $\left.\mathrm{CH}\left(\mathrm{C}_{2} \mathrm{H}_{5}\right) \mathrm{CO}_{2}\right]_{2} \mathrm{Sn}\left(\mathrm{SnOct}_{2}\right)$ foram fornecidos pela Aldrich. Poli(glicol etilênico) (PEG), Mn =600 (178-187 mg KOH/g) (líquido) e $\mathrm{Mn}=4000$ (25-31 mg KOH/g) (sólido) foram fornecidos pela Oxiteno do Brasil. Clorofórmio, metanol, hexano, tetra-hidrofurano (THF) e acetona foram fornecidos pela Synth (Brasil). Todos os materiais foram utilizados sem purificação adicional.

Síntese dos copolímeros PLA-PEG-PLA: as duas séries de copolímeros foram sintetizadas mantendo uma razão préestabelecida entre os blocos hidrofóbico e hidrofílico, ou seja, razões molares calculadas, LA:EO, entre os meros lactato (O$\left.\mathrm{CH}\left(\mathrm{CH}_{3}\right)-\mathrm{CO}\right)$ e óxido de etileno $\left(\mathrm{CH}_{2}-\mathrm{CH}_{2}-\mathrm{O}\right)$. Foram preparados copolímeros com as seguintes composições LA:EO na alimentação: $16,6: 83,4(0,2) ; 33,3: 66,6(0,5) ; 41,2: 58,8$ $(0,7) ; 50: 50(1,0)$ e $66,6: 33,3(2,0)$. A polimerização foi conduzida com pequenas modificações, de acordo com métodos previamente relatados ${ }^{[15]}$. Por exemplo: em câmara com atmosfera controlada, purgada com nitrogênio seco, quantidades de $\mathrm{PEG}$ e $\mathrm{SnOct}_{2}$ previamente pesadas, na proporção de 0,0124 mol de $\mathrm{SnOct}_{2}$ por mol de hidroxila do PEG, foram introduzidas em um tubo seco. $\mathrm{O}$ tubo foi selado e imerso em banho de óleo de silicone a $120{ }^{\circ} \mathrm{C}$ por dez minutos, e então resfriado à temperatura ambiente. Uma massa préestabelecida de 1,1-lactídeo, foi então introduzida no tubo sob atmosfera de nitrogênio. Os tubos foram novamente selados e imersos em banho a $120^{\circ} \mathrm{C}$ por 22 horas. O copolímero assim obtido foi purificado por dissolução em clorofórmio e precipitação em metanol (três vezes). Os copolímeros no estado líquido foram separados do meio por precipitação em metanol a $0{ }^{\circ} \mathrm{C}$. O copolímero foi filtrado e seco a temperatura ambiente e sob pressão reduzida por 24 horas.

Três diferentes conjuntos (A, B e C) (Tabela 1) de condições reacionais foram estudados. Nos conjuntos A e B, a temperatura e o tempo de reação foram $120^{\circ} \mathrm{C}$ e 22 horas com uma concentração de catalisador de 0,0038 ou $0,0124 \mathrm{~mol}$ por mol de grupo hidroxila, respectivamente. No conjunto $\mathrm{C}$ a temperatura e o tempo de reação foram aumentados para $135{ }^{\circ} \mathrm{C}$ e 89 horas com uma concentração de catalisador de 1,0 mol por mol de grupo hidroxila. Diferentes tamanhos de bloco PLA foram obtidos no copolímero, de acordo com a variação da massa relativa de 1,1-lactídeo na alimentação, como mostrado na Tabela 1. O homopolímero PLA foi sintetizado seguindo o procedimento descrito acima sem a adição de coiniciador PEG, utilizando a razão molar 1-lactideo:SnOct ${ }_{2}$ igual a 878:1 e um tempo total de reação de 5 horas. 
Tabela 1: Composições calculadas e obtidas nos copolímeros

\begin{tabular}{|c|c|c|c|c|c|c|c|}
\hline Copolímero & \multicolumn{2}{|c|}{ Alimentação } & \multicolumn{5}{|c|}{ Copolímero } \\
\hline \multirow{6}{*}{$\begin{array}{c}\text { PEO } 600 \\
\text { (A) }\end{array}$} & & LA:EO & $\mathrm{DP}_{\mathrm{PLA}}$ & $\mathrm{LA}: \mathrm{EO}^{\mathrm{a}}$ & $\mathrm{DP}_{\mathrm{PLA}}{ }^{\mathrm{b}}$ & $\mathrm{Mn}^{\mathrm{c}}$ & Aspecto \\
\hline & 1 & 0,2 & 1,4 & 0,17 & 1,2 & 773 & líquido \\
\hline & 2 & 0,5 & 3,4 & 0,49 & 3,3 & 1075 & líquido \\
\hline & 3 & 0,7 & 4,8 & 0,67 & 4,6 & 1262 & líquido \\
\hline & 4 & 1,0 & 6,8 & 1,00 & 6,8 & 1579 & ceroso \\
\hline & 5 & 1,3 & 8,8 & 1,30 & 8,9 & 1882 & ceroso \\
\hline \multirow{5}{*}{$\begin{array}{l}\text { PEO4000 } \\
\text { (B) }\end{array}$} & 6 & 0,2 & 9,1 & 0,15 & 6,8 & 4979 & Sólido \\
\hline & 7 & 0,5 & 22,8 & 0,35 & 15,9 & 6290 & Sólido \\
\hline & 8 & 0,7 & 31,9 & 0,79 & 35,9 & 9170 & Sólido \\
\hline & 9 & 1,0 & 45,5 & 1,27 & 57,8 & 12323 & Sólido \\
\hline & 10 & 2,0 & 91 & 3,20 & 145,6 & 24966 & Sólido \\
\hline \multirow{5}{*}{$\begin{array}{l}\text { PEO4000 } \\
\text { (C) }\end{array}$} & 11 & 0,2 & 9,1 & 0,14 & 6,4 & 4922 & Sólido \\
\hline & 12 & 0,5 & 22,8 & 0,34 & 15,5 & 6232 & Sólido \\
\hline & 13 & 0,7 & 31,9 & 0,57 & 25,9 & 7730 & Sólido \\
\hline & 14 & 1,0 & 45,5 & 0,66 & 30,0 & 8320 & Sólido \\
\hline & 15 & 2,0 & 91 & 1,65 & 75,1 & 148,1 & Sólido \\
\hline
\end{tabular}

${ }^{a}$ Razão LA:EO no copolímero determinada por $R M N-{ }^{1} H$ e Equação $1 ;{ }^{b}$ Grau de polimerizaçao do PLA, $D P_{P L A}=D P_{\text {PEO }} \times\left(L A: E O^{a}\right) / 2 ;{ }^{c} P e s o$ molecular médio numérico, $\mathrm{Mn}=\left(44 \times D P_{\mathrm{PEO}}\right)+\left(144 \times D P_{\mathrm{PLA}}\right)$

\section{Caracterização dos copolímeros triblocos}

Teste de solubilidade: Os materiais sintetizados foram testados em água, etanol, THF e $\mathrm{CHCl}_{3}$. Os resultados foram comparados aos obtidos a partir dos homopolímeros PLA e PEG correspondentes.

Espectroscopia no infravermelho (FTIR): A técnica de FTIR foi utilizada para caracterizar a ligação entre os blocos PLA e PEG. Os espectros de filmes poliméricos sobre placa de $\mathrm{KBr}$, obtidos de soluções em clorofórmio, foram analisados no modo transmissão usando um espectrofotômetro Nicolet Magma-IR 560 (FTIR), equipado com detector DTGS-KBr.

Espectroscopia de Ressonância Magnética Nuclear: Espectros de RMN- ${ }^{1} \mathrm{H}$ foram obtidos a $200 \mathrm{MHz}$ a partir de soluções de clorofórmio $\left(\mathrm{CDCl}_{3}\right)$ usando um espectrômetro Bruker AC-200 e tetrametilsilano (TMS) como referência interna. Espectros de $\mathrm{RMN}-{ }^{1} \mathrm{H}$ em $\mathrm{CDCl}_{3}$ foram empregados para determinar a razão molar LA:EO no copolímero e estimar a composição relativa. A razão molar no copolímero LA:EO foi determinada pela Equação 1.

$$
\frac{L A}{E O}=\frac{\frac{a}{3}}{\frac{e}{4}}=1,333 \times \frac{a}{e}
$$

Onde: a =Integração da área correspondente ao grupo metila do PLA (1,4-1,6 $\delta)$.

$\mathrm{e}=$ Integração da área correspondente ao grupo metileno do PEG $(3,4-3,7 \delta)$

Análise termogravimétrica (TG): Os ensaios foram realizados usando um equipamento TG-DTA Simultâneo, modelo SDT 2960, da TA Instruments, a uma taxa de aquecimento de $10{ }^{\circ} \mathrm{C} / \mathrm{min}$. e faixa de temperatura de 25 a $800^{\circ} \mathrm{C}$, em atmosfera de nitrogênio.

Calorimetria exploratória diferencial (DSC): Ensaios foram realizados usando o equipamento Mettler Toledo 822 sob atmosfera de nitrogênio. As amostras com massas entre 10 e $12( \pm 0,1) \mathrm{mg}$ foram encapsuladas em porta-amostras de alumínio e submetidas a ciclos de aquecimento na faixa de -70 a $120{ }^{\circ} \mathrm{C}$, a uma taxa de $10{ }^{\circ} \mathrm{C} / \mathrm{min}$.

\section{Resultados e Discussão}

Os graus de polimerização $\left(\mathrm{DP}_{\mathrm{PLA}}\right)$ do bloco PLA dos copolímeros preparados neste trabalho são mostrados na Tabela 1.

Nas reações de 1 a 5, a partir do PEG 600, a mistura permaneceu homogênea e transparente durante o transcorrer da reação. Nas reações de 6 a 15, cinco minutos após a imersão 
em banho a $120^{\circ} \mathrm{C}$ a mistura reacional contendo PEG 4000 e 1-lactideo sólidos transformou-se em líquido transparente. Com o prosseguimento da reação são formados segmentos mais longos de PLA, e a mistura reacional tornou-se progressivamente mais viscosa e turva, ocorrendo separação de fase decorrente da insolubilidade dos copolímeros de massa molar mais elevada, após duas horas de reação, aproximadamente. No conjunto reacional $\mathrm{C}$ ocorreu a formação de um precipitado branco durante a polimerização. A formação deste subproduto foi atribuída à degradação do $\mathrm{SnOct}_{2}$ utilizado em excesso ( $1 \mathrm{~mol} \mathrm{SnOct}_{2}$ por mol de hidroxila). O precipitado branco foi analisado quanto à sua solubilidade e revelou-se insolúvel em água e em solventes orgânicos usuais, polares e apolares. O aspecto dos copolímeros a partir de PEG 600 variou de líquido a sólido, com o acréscimo da razão LA:EO na alimentação. Esta variação de aspecto sugere que diferentes tamanhos de blocos PLA foram ligados ao pré-polímero PEG 600 líquido inicialmente.

Análises comparativas de solubilidade sugerem a formação do copolímero. O homopolímero PEG mostrou-se solúvel em água, etanol, THF e $\mathrm{CHCl}_{3}$, enquanto o PLA foi solúvel somente em $\mathrm{CHCl}_{3}$. O produto final da reação mostrou um comportamento de solubilidade intermediário aos segmentos PLA e PEG constituintes, formou-se dispersão (emulsão turva) com água, etanol e THF, e solução homogênea em $\mathrm{CHCl}_{3}$.

Espectros FTIR do PLA, PEG-4000 e do copolímero 1 são mostrados na Figura 1. As absorções características do PLA são três fortes bandas devidas a vibrações do grupo C-CO-O-C. Ou seja, a banda devida ao estiramento do $\mathrm{C}=0 \mathrm{em} 1751 \mathrm{~cm}^{-1}$, a banda devida ao estiramento assimétrico do C-O em 1195 $\mathrm{cm}^{-1}$ e em $1110 \mathrm{~cm}^{-1}$ ao estiramento simétrico C-O-C. A ausência de uma banda intensa na região $3500-3000 \mathrm{~cm}^{-1}$ (estiramento do grupamento $0-\mathrm{H}$ ) é um indicativo de ausência de subprodutos de hidrólise do PLA. Para o PEG, as absorções características são uma banda larga e intensa devido aos grupos
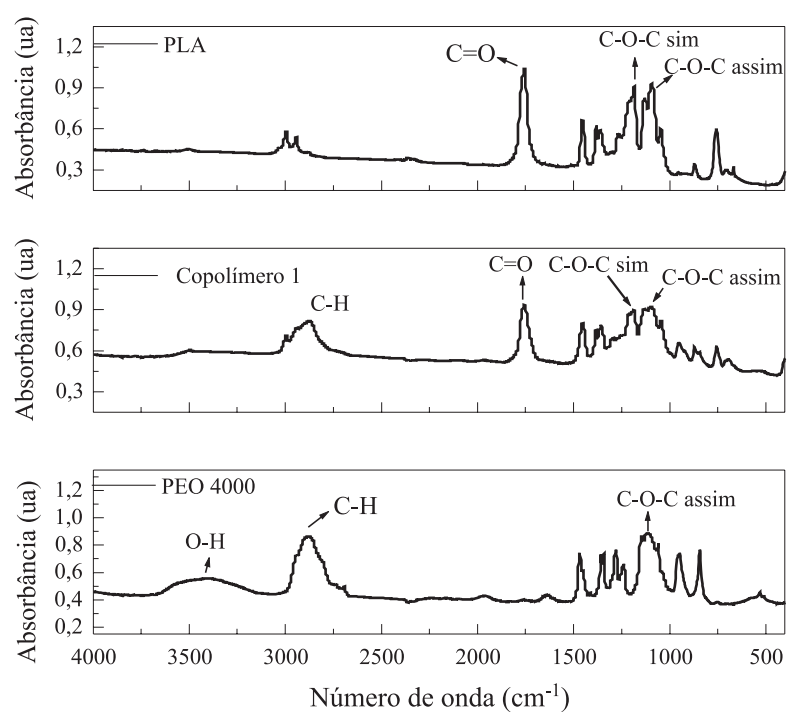

Figura 1: Espectros FTIR do copolímero 1 e dos homopolímeros PLA e PEO-4000 hidroxila terminais da cadeia do PEG associados por pontes de hidrogênio $\left(3400 \mathrm{~cm}^{-1}\right)$, e uma banda intensa em $1110 \mathrm{~cm}^{-1}$ devida ao estiramento assimétrico C-O-C .

No espectro do copolímero, a banda em $3400 \mathrm{~cm}^{-1}$ aparece reduzida, mostrando que o homopolímero precursor, $\mathrm{PEG}$, foi modificado, as bandas em $1739 \mathrm{~cm}^{-1}$ e $1110 \mathrm{~cm}^{-1}$ são atribuídas ao estiramento do grupo carbonila $(\mathrm{C}=\mathrm{O})$ e ao estiramento assimétrico do grupo (C-O-C). As características intermediárias deste espectro, em relação aos blocos constituintes PLA e PEG, são relevantes e sugerem a formação do copolímero. Esta técnica não permite afirmar que os segmentos PEG e PLA estão unidos por ligação covalente resultante da poliesterificaçao, e que os produtos formados não são apenas misturas poliméricas, entretanto corrobora resultados de outras técnicas (solubilidade, HNMR e DSC) empregadas para a caracterização da estrutura química do produto obtido e descritas mais adiante.

Análises detalhadas dos espectros $\mathrm{RMN}-{ }^{1} \mathrm{H}$ permitiram a caracterização da ligação entre os blocos PLA-PEG, e a determinação quantitativa do grau de polimerização do bloco PLA. A estrutura do copolímero e o espectro do copolímero 8, dos homopolímeros PLA e PEG são mostrados na Figura 2. Os valores de deslocamentos químicos observados para o copolímero estão de acordo com os relacionados em literatura ${ }^{[16]}$.

No espectro do copolímero, Figura 2, o multipleto em 4,1-4,3 $\delta$ (atribuído a ambos os prótons e' + b') é atribuído aos prótons metilenos alfa terminais da cadeia do bloco PEG (PLA-COO$\mathrm{CH}_{2}$ ), e', e aos prótons metinos finais de cadeia dos blocos PLAs, b'. A presença deste multipleto é um indicativo da ligação PLAPEG. O pico correspondente a b', deveria estar presente no espectro do homopolímero PLA, entretanto não é observado provavelmente devido a sua baixa intensidade.

Na Tabela 1 é mostrada a composição estimada em comparação a composição obtida no copolímero. O grau de

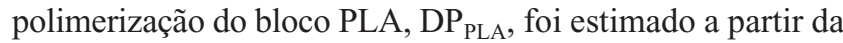

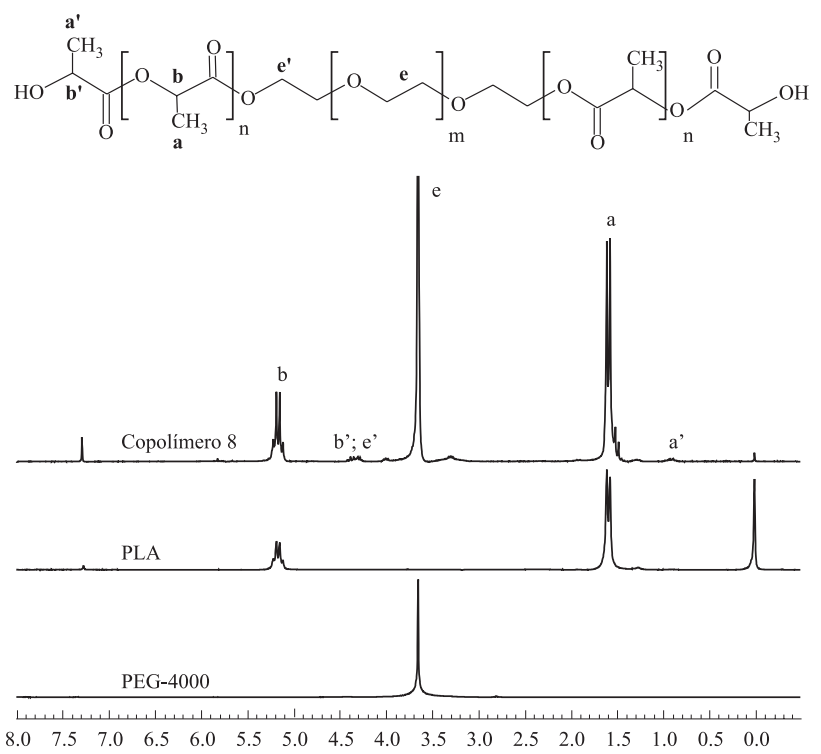

Figura 2: Estrutura química do copolímero tribloco e espectros de RMN${ }^{1} \mathrm{H}$ do copolímero 8 e dos homopolímeros PLA e PEG-4000 
razão LA:EO determinada por $\mathrm{RMN}-{ }^{1} \mathrm{H}$ e do grau de polimerização do polímero precursor $\mathrm{PEG}$, ou seja, o estimado $\mathrm{DP}_{\mathrm{PLA}}$ no copolímero foi $\mathrm{DP}_{\mathrm{PEG}} \mathrm{X}(\mathrm{LA} / \mathrm{EO}) / 2$.

O PEG 600 apresentou uma conversão quase completa (Tabela 1); quase todo 1,1-lactídeo alimentado foi incorporado às cadeias do pré-polímero PEG. Para PEG 4000 (conjunto $\mathrm{B}$ e C) os reagentes alimentados foram apenas parcialmente convertidos a copolímeros, e o $\mathrm{DP}_{\mathrm{PLA}}$ variou do valor projetado (alimentado). Este comportamento pode ser atribuído às condições de polimerização usadas. $\mathrm{Na}$ poliadição via inserção por coordenação, o crescimento das cadeias ocorre simultaneamente ao longo da reação. Assim, altas massas molares são alcançadas apenas a altas conversões. A técnica de preparação usada, polimerização em massa, emprega como solvente o próprio reagente (monômero/ polímero precursor) sem diluentes. Em geral, durante a reação, a viscosidade do meio reacional cresce rapidamente dificultando a difusão dos reagentes necessária ao crescimento das cadeias. Na síntese a partir do PEG 600, o copolímero projetado possuía massa molar menor e, portanto, permanece solúvel no sistema reacional durante toda a reação. Ao contrário, copolímeros a partir do PEG 4000 possuíam maior massa molar projetada, e solidificaram após a primeira hora de reação separando-se do meio reacional. Assim, enquanto os copolímeros a partir de PEG 600 foram sintetizados com uma composição quantitativa quase idêntica à projetada, os copolímeros a partir do PEG 4000 apresentaram desvio da composição projetada.

\section{Análise térmica das amostras: PEG600; PEG4000; copolímeros 3 e 8}

Análises termogravimétricas (TG) dos copolímeros $3 \mathrm{e}$ 8 foram realizadas para obter informações sobre a natureza das interações presentes no copolímero por meio de medidas de estabilidade térmica e de cinética de degradação. $\mathrm{Na}$ Figura 3 são apresentadas as curvas representativas das análises termogravimétricas dos homopolímeros e dos copolímeros em bloco.

Para a amostra PEG 4000, a curva de termogravimetria (TG) (Fig. 3a) mostra um único estágio de decomposição de $\mathrm{T}_{\mathrm{i}}=370^{\circ} \mathrm{C}$ e $\mathrm{T}_{\mathrm{f}}=430^{\circ} \mathrm{C}$ com uma perda de massa de aproximadamente $98 \%$ e um resíduo de $2 \%$, sugerindo um composto orgânico bem puro. A curva da termogravimetria derivada (DTG) confirma essa decomposição principal, mas são observadas duas etapas de degradação, o que corrobora a curva de DTA, que mostra também outro evento endotérmico abaixo de $100^{\circ} \mathrm{C}$, indicando possível perda de solvente ou água.

Para o copolímero 8, a curva de TG (Fig.3b) mostra dois estágios de decomposição, o primeiro a $\mathrm{T}_{\mathrm{i}}=200^{\circ} \mathrm{C}$ com uma perda de massa de $16 \%$ e um segundo estágio de decomposição, que é o principal, a $\mathrm{T}_{\mathrm{i}}=372^{\circ} \mathrm{C}$ com uma perda de massa de $78 \%$, e por fim um resíduo de $2 \%$ foi observado. A curva de DTG confirma dois estágios de degradação. A curva de DTA confirma esses dois eventos e mostra também um terceiro evento endotérmico com temperatura abaixo de $100^{\circ} \mathrm{C}$, referente à perda de solvente ou água.

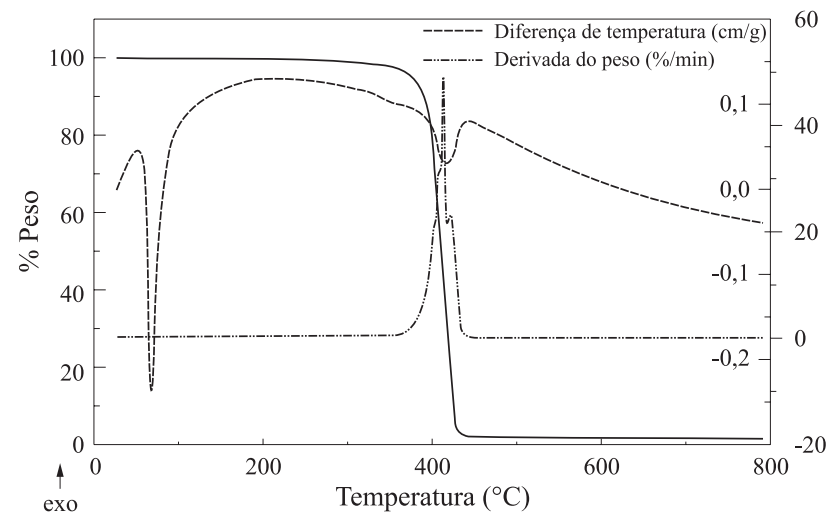

Figura 3a. Curvas de TG-DTA do PEG-4000

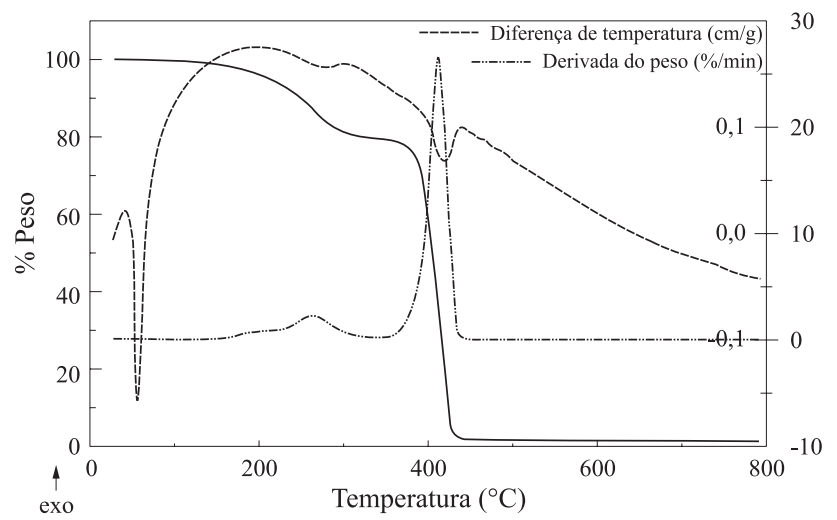

Figura 3b. Curvas de TG-DTA do copolímero 8

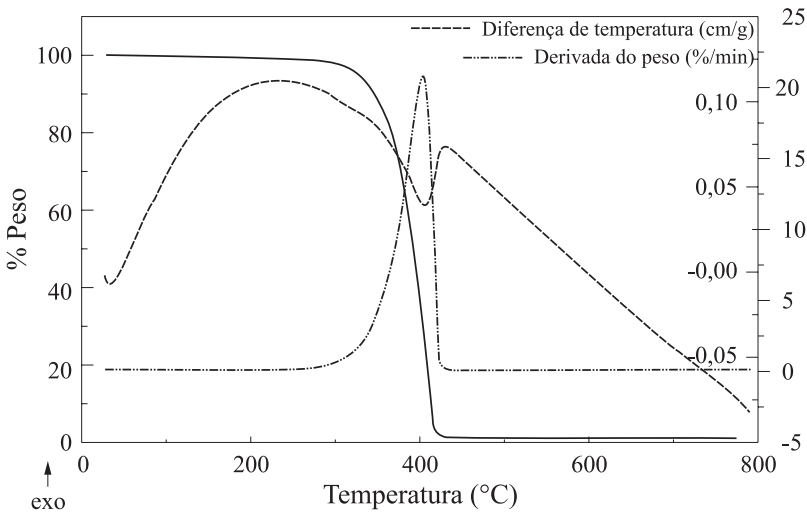

Figura 3C. Curvas de TG-DTA do PEG-600

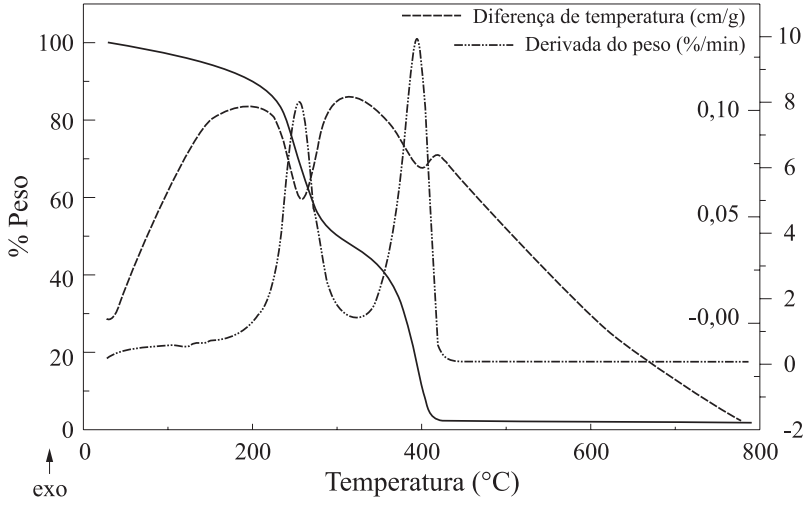

Figura 3d. Curvas de TG-DTA do copolímero 3 


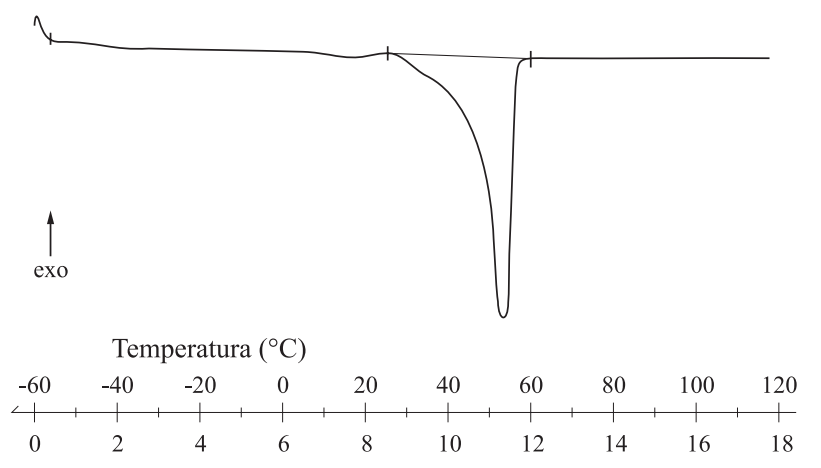

Figura 4. Curva de DSC do copolímero 8

Para a amostra PEG 600, a curva de TG (Fig 3c) mostra um único estágio de decomposição a $\mathrm{T}_{\mathrm{i}}=300^{\circ} \mathrm{C}$ com uma perda de massa de aproximadamente $100 \%$, não deixando resíduo, ou seja com decomposição total do polímero. O comportamento observado foi confirmado pela curva da DTG com uma velocidade máxima de decomposição a $400{ }^{\circ} \mathrm{C}$, a mesma temperatura do evento endotérmico apresentado na curva de DTA.

Para o copolímero 3, a curva de TG (Fig. 3d) apresentou dois estágios de decomposição nas temperaturas $\mathrm{T}_{\mathrm{i}}=80^{\circ} \mathrm{C}$ (para o primeiro estágio) e $\mathrm{T}_{\mathrm{i}}=350^{\circ} \mathrm{C}$ (para o segundo), porém em ambos estágios a degradação apresentou-se lenta comparada com a observada para o copolímero 8 (Fig. 3b), que transcorreu em faixas mais estreitas de temperatura. A curva de DTG confirma esses dois estágios e mostrou que a velocidade máxima para o primeiro estágio foi a $250{ }^{\circ} \mathrm{C}$ e para o segundo a $390{ }^{\circ} \mathrm{C}$, ambos confirmados pelos dois eventos endotérmicos da curva de DTA. Essas análises sugerem que esse composto apresentou a menor estabilidade térmica e o menor grau de pureza.

Ambos os copolímeros apresentaram dois estágios de degradação térmica e decréscimo de estabilidade térmica quando comparados aos respectivos homopolímeros PEG originais, $M n=600$ ou 4000 . No primeiro estágio ocorreu a degradação a partir das terminações das cadeias dos blocos PLAs, favorecida pela formação de éster cíclico lactídeo, e no segundo estágio, a alta temperatura, ocorreu a cisão térmica da cadeia polimérica. O comportamento térmico foi previamente relatado para polímeros amorfos preparados a partir de $(\mathrm{d}, 1)$ lactídeo e seus copolímeros ${ }^{[17-18]}$.

Uma única $\mathrm{T}_{\mathrm{g}} \mathrm{a}-40{ }^{\circ} \mathrm{C}$, intermediária às $\mathrm{T}_{\mathrm{g}}$ do $\mathrm{PEG}$ $\left(-70{ }^{\circ} \mathrm{C}\right)$ e do PLA $\left(65^{\circ} \mathrm{C}\right)$, pode ser observada no DSC do copolímero 8 (Figura 4), indicando uma miscibilidade entre as regiões amorfas dos blocos PLA e PEG-4000. Um único pico endotérmico é observado em $\mathrm{T}_{\mathrm{m}}=56^{\circ} \mathrm{C}$ e é atribuído à fusão cristalina do bloco PLA indicando que a cristalização do bloco PEG foi suprimida no copolímero.

\section{Conclusões}

Copolímeros anfifílicos PLA-PEG-PLA foram obtidos pela ciclo-polimerização de 1,1-lactídeo utilizando di-2-etil-hexanoato de estanho como iniciador e o pré-polímero poli(glicol etilênico) di-hidroxi terminado ( $\mathrm{Mn}=600$ ou 4000) como co-iniciador. A formação dos copolímeros foi caracterizada quanto à estrutura química e propriedades físico-químicas por espectroscopia no infravermelho e espectrometria de ressonância magnética de hidrogênio, análise termogravimétrica, análise térmica diferencial e calorimetria exploratória diferencial.

A diversidade de aspecto (líquido à sólido) dos copolímeros, controlado pela massa molar do co-iniciador PEG e pela razão LA:EO, os torna bastante adaptáveis a vasta gama de aplicações.

Concentrações de $1 \mathrm{~mol} \mathrm{Sn}(\mathrm{Oct})_{2}$ por mol de grupo hidroxila, ou maior, em reação de polimerização se mostrou desproporcional e deletério.

\section{Agradecimentos}

Os autores agradecem à FAPESP (99/01783-0), à CAPES e ao CNPq (milênio) pelos apoios financeiros.

\section{Referências Bibliográficas}

1. Kricheldorf, H. R. - Chemosphere, 43, p.49 (2001).

2. Middleton, J. C. - Biomaterials, 21, p.2335 (2001).

3. Lunt, J. - Degradation and Polymer Stability, 59, p.145 (1998).

4. Sawhney, A. S., Pathak, C.P., Hubbell, J. A. - Macromolecules, 26, p.581 (1993)

5. Pietrzak, W. S., Sarver, D., Verstynen, M. - Bone, 19, p.109s (1996)

6. Heald, C. R., Stolnik, S., De Matteis, C., Leermakers F.A.M. Colloids and surfaces A: Physicochemical and Engineering Aspects, 179, p.79 92001)

7. Kim S. Y., Shin, I. G., Lee, Y. M. - Journal of Controlled Release, 56, p.197 (1998)

8. Chony, M., Fishbein, I., Danenberg, H. D., Golomb, G. - Journal of Controlled Release, 83, p.389 (2002)

9. Matsumoto, J., Nakada, Y., Sakurai K., Nakamura T., Takahashi, Y. - International Journal of Pharmaceuties, 185, p.93 (1999)

10. Torchilin, V. P. - Journal of Controlled Release, 73, p.137 (2001)

11. Ryner, M., Stridsberg, K., Alberson, A., Schenck, H., Svensson, M. - Macromolecules, 34, p.3877 (2001)

12. Kricheldorf, H. R., Saunders, I. K., Boettcher C. - Polymer, 36 , p.1253 (1995).

13. Kowalski, A., Duda, A., Penczek,S. - Macromolecules, 33, p.7359 (2000)

14. Stridsberg, K. M., Ryner, M., Albertson, A. C. - Adv. Polym. Sci., 157, p.41 (2002).

15. Dong, C., Qiu, K. Y., Gu, Z. W., Feng, X. D. - Macromolecules, 34, p.4691 (2001).

16. Du, Y. j., Lemstra, P. J., Nijenhuis, A. J. - Macromolecules, 28, p.2124 (1995).

17. Penco, M., Bignotti, F., Sartore, S., D’Antone, S, D'Amore, A. - J. Appl. Polym. Sci., 78, p. 1721 (2000).

18. Khabbaz, F., Karlsson, S., Albertsson, A.C. - J. Appl. Polym. Sci., 78 p.2369 (2000).

Enviado: $26 / 11 / 03$

Reenviado: $27 / 02 / 04$

Aprovado: 15/03/04 